\title{
Original Article \\ Hepatitis A and E Outbreak Surveillance during 2015-2017 in Kashmir, India: Is the Water to Blame?
}

\author{
Syed Manzoor Kadri ${ }^{1}$, Saleem-ur-Rehman², Kausar Rehana ${ }^{3}$, Despoina-Rafailia Benetou ${ }^{4}$, Dar Farooz Ahmad ${ }^{5}$, \\ Afshan Abdullah ${ }^{6}$, Vijay Kumar Chattu** \\ ${ }^{1}$ Department of Communicable Diseases, Kashmir, India \\ ${ }^{2}$ Directorate of Health Services, Kashmir, India \\ ${ }^{3}$ Division of Epidemiology, Directorate of Health Services, Kashmir, India \\ ${ }^{4}$ University of Athens, Athens, Greece \\ ${ }^{5}$ Integrated Disease Surveillance Programme, Kashmir, India \\ ${ }^{6}$ Division of Epidemiology and Public Health, Integrated Disease Surveillance Programme, Barzulla, Kashmir, India \\ ${ }^{7}$ Department of Paraclinical Sciences, Faculty of Medical Sciences, The University of the West Indies, St. Augustine, Trinidad and Tobago
}

\section{ARTICLE INFO}

\section{Article History}

Received 22 March 2018

Accepted 28 August 2018

Keywords

Fecal-oral route

Hepatitis A

Hepatitis E

sanitary measures

water contamination

\begin{abstract}
Waterborne diseases, such as Hepatitis A and E, are major public health concerns in most of the developing nations, indicating the need for proper outbreak prevention, surveillance, and timely management. This study presents data regarding the prevalence and epidemiological characteristics of hepatitis A and E outbreaks as well as water quality in Kashmir, India, during 2015-2017. Hepatitis outbreaks were initially investigated by rapid response teams, under the Integrated Disease Surveillance Programme. Suspected cases were further evaluated by blood sampling to confirm the disease along with water sampling evaluation. Between 2015 and 2017, 23 disease outbreaks were recorded; among these, four outbreaks occurred in 2015, 12 in 2016, and seven in 2017. Specifically, 12 of the total outbreaks were concerned with hepatitis A infection, 10 concerned hepatitis $E$ infection, and one involved eight cases of jaundice with neither hepatitis A virus nor hepatitis A virus detected in blood sampling. Overall, during the aforementioned period, 393 cases of hepatitis A or E were detected. Regarding water quality, which was evaluated using the most probable number method for counting coliform, 38 of 50 water samples were found to be unfit for human consumption and one source was found to be suspicious. This study of prevalence and epidemiology of hepatitis A and E along with its relationship with water quality and socioeconomic factors, such as poor hygiene and lack of access to safe water, aids towards the implementation of effective preventive sanitary measures and public health actions.
\end{abstract}

(C) 2018 Atlantis Press International B.V. This is an open access article under the CC BY-NC license (http://creativecommons.org/licenses/by-nc/4.0/)

\section{INTRODUCTION}

Although water is one of the essential elements for maintaining life, it can also serve as a medium for hazardous substances and pathogenic microorganisms, thus posing substantial health threats to humans [1]. Waterborne diseases, especially infectious ones, such as hepatitis A and E, are a huge concern for developing countries, such as India, which suffer from water-related outbreaks due to the lack of hygienic and sanitary environment [2,3]. Lack of access to safe water and water sanitation are the major contributors to water-associated infections in India despite long-standing efforts by the government and society to improve water hygiene and supply [2]. Both hepatitis A (HAV) and hepatitis E (HEV) are viral infections that are transmitted through the ingestion of food and water contaminated with fecal matter of an infected person. Worldwide, an estimated 12-15 million acute cases of hepatitis A, resulting in 3000 deaths, and an estimated 14 million symptomatic cases of hepatitis E, resulting in 300,000 deaths and 5200 stillbirths, are reported annually [4]. These numbers indicate the great need

\footnotetext{
*Corresponding author. Emails: vijay.chattu@sta.uwi.edu; vkchattu@gmail.com
}

for disease prevention, which mainly includes vaccination (for HAV infection), water sanitation, and proper personal hygiene. Incidence rates of acute $\mathrm{HAV}$ and $\mathrm{HEV}$ infections are relatively high in India, where the outbreaks are considered epidemic [2]. The incidence of HAV is higher in infants and young adults compared with elderly individuals in India; by contrast, HEV affects older children and adults worldwide and is considered common in the majority of developing countries, especially tropical areas [3]. Concerning the prevalence of viral hepatitis in India, the Integrated Disease Surveillance Programme (IDSP) presented a report on morbidity and mortality resulting from hepatitis A and E viral infections between 2011 and 2013, which concluded that the burden of viral hepatitis in India is not well characterized [5]. During the 3-year period, 804,782 hepatitis cases and 291 outbreaks across India were reported to the IDSP. Among 599,605 cases tested for hepatitis A, 44,663 (7.4\%) were positive, and among 187,040 tested for hepatitis E, $19,508(10.4 \%)$ were positive. Worryingly, two-thirds of outbreaks were reported from rural areas [5]. Among 163 (56\%) outbreaks with known etiology, 78 (48\%) were caused by hepatitis E, $54(33 \%)$ by hepatitis A, and $19(12 \%)$ by both hepatitis A and $\mathrm{E}$ [5]. Contaminated drinking water was identified as a cause for 
$72 \%(109 / 151)$ of the hepatitis A and E outbreaks, and was implicated in 49 (38\%) of the 128 outbreaks for which laboratory confirmation was not available [5].

The reasons behind major viral hepatitis outbreaks in India are related to the country's socioeconomic status and development. First, contaminated water sources are the most commonly reported cause of hepatitis A and E outbreaks, highlighting the lack of access to safe and clean water supply to several parts of the country, especially in rural areas. Second, low rates of vaccination coverage for hepatitis A, again, especially in rural areas, as a result of the unavailability or inaccessibility of the vaccine contribute to the relatively high incidence of hepatitis A cases. Therefore, it has been made clear that routine surveillance of waterborne diseases is a core public health concern to prevent infectious diseases, improve population health and well-being, and to ensure safe and sanitary living conditions. Further investigation and studies regarding humanistic as well as economic burden of HAV and HEV infections should be performed on a national level to inform and help policymakers make appropriate decisions regarding public health policies, effectiveness of water sanitation, and immunization for disease prevention. Thus, our study aims to provide epidemiological data on hepatitis A and E cases, registered as part of the IDSP, along with the evaluation and rating of water samples, in different areas of Kashmir between 2015 and 2017.

\section{MATERIALS AND METHODS}

\subsection{Study Design}

This was a cross-sectional study that involved analysis of data routinely reported as part of the IDSP. During the past decade, the IDSP has been in charge of reporting and managing outbreaks of communicable diseases across different districts of India, providing surveillance for disease trends as well as disease seasonality.

\subsection{Setting}

The study was conducted in Kashmir Division in the state of Jammu and Kashmir (North India), and included all of the districts in the Kashmir region (Anantnag, Bandipora, Baramulla, Budgam, Ganderbal, Kulgam, Kupwara, Pulwama, Shopian, and Srinagar).

\subsection{Study Population and Study Period}

The study was conducted among Kashmiris in India. From 2015 to 2017, IDSP collected data on hepatitis A and E outbreaks in various districts, giving insights into their incidence rates and epidemiological characteristics across the country.

\subsection{Data Collection Procedure}

If a cluster of cases with high suspicion of the illness (hepatitis A or E), such as symptoms of jaundice, anorexia, and hepatomegaly, was suspected in any area, it was first investigated by the rapid response teams to evaluate and investigate a possible outbreak. Laboratory investigations, including blood and water sample collection and testing, were then performed on a sample of suspected cases, as well as on the water facilities in that region, to establish the diagnosis, which requires increased level of bilirubin and $>2.5$ times the upper value of serum enzymes alanine aminotransferase and aspartate transaminase, and acknowledge any possible contaminated water source (Figs. 1A and 1B). Specifically, serum samples were tested for immunoglobulin M (IgM) anti-HAV and IgM anti-HEV antibodies for the detection of acute hepatitis A and acute hepatitis E, respectively, using commercially available enzyme-linked immunosorbent assay kits (Dia.Pro Diagnostic Bioprobes srl, Milan, Italy). Water quality testing involved the collection, labeling, and transportation of about $250 \mathrm{~mL}$ of water samples to the laboratory for bacteriological analysis. The most probable number (MPN) method for counting coliform was used as the water quality monitoring technique to detect fecal contamination. The water quality was then rated as follows: excellent $(\mathrm{MPN}=0)$, satisfactory $(\mathrm{MPN}=1-3 / 100 \mathrm{~mL})$, suspicious $(\mathrm{MPN}=4-10 / 100 \mathrm{~mL})$, or unsatisfactory (MPN > 10/100 mL).

\subsection{Data Entry and Analysis}

Following data collection, further analysis was performed using Microsoft Excel (Microsoft, Redmond, WA, USA) and SPSS (IBM, New York, NY, USA). Appropriate actions were undertaken by the respective state/district surveillance units (Fig. 2).

\subsection{Ethics}

The ethical approval was given by the Director of Health Services, Kashmir, India.

\section{RESULTS}

During the 3-year period (2015-2017) of hepatitis A and E surveillance and registration, 23 outbreaks were detected, as depicted in Fig. 3, which affected the districts of Anantnag, Baramulla, Budgam, Ganderbal, Kulgam, Kupwara, and Pulwama. A total of 393 cases of hepatitis were reported. Specifically, 12 of the outbreaks involved hepatitis A infection, 10 involved hepatitis $\mathrm{E}$ infection, and one involved eight cases of jaundice with neither HAV nor HEV detected in blood samples. Genotyping of HAV or HEV was not available in any of the confirmed hepatitis cases. Detailed descriptions of each outbreak along with epidemiological data are summarized in Table 1.

In 2015, two outbreaks of each hepatitis A and E were observed. Both hepatitis A outbreaks and one of the hepatitis E outbreaks affected the district of Baramulla, specifically the areas of Hanjiwara Bala, Barzulla, and Dangiwacha, whereas the other hepatitis E outbreak was recorded in the district of Kulgam, specifically in Nipora and Qazigund. A total of 141 (91 males, 50 females) suspected cases (83 cases of hepatitis A and 58 cases of hepatitis E) were reported, and 41 blood samples were drawn, of which 26 were found to be positive for either HAV or HEV. The seropositivity rate for hepatitis A was 73\% (19/26 blood samples tested positive for HAV), whereas that for hepatitis E was 47\% (7/15 blood samples tested positive for HEV). During water quality testing, 12 water samples were evaluated, of which 10 were deemed to be unsatisfactory. The other two had satisfactory quality. 

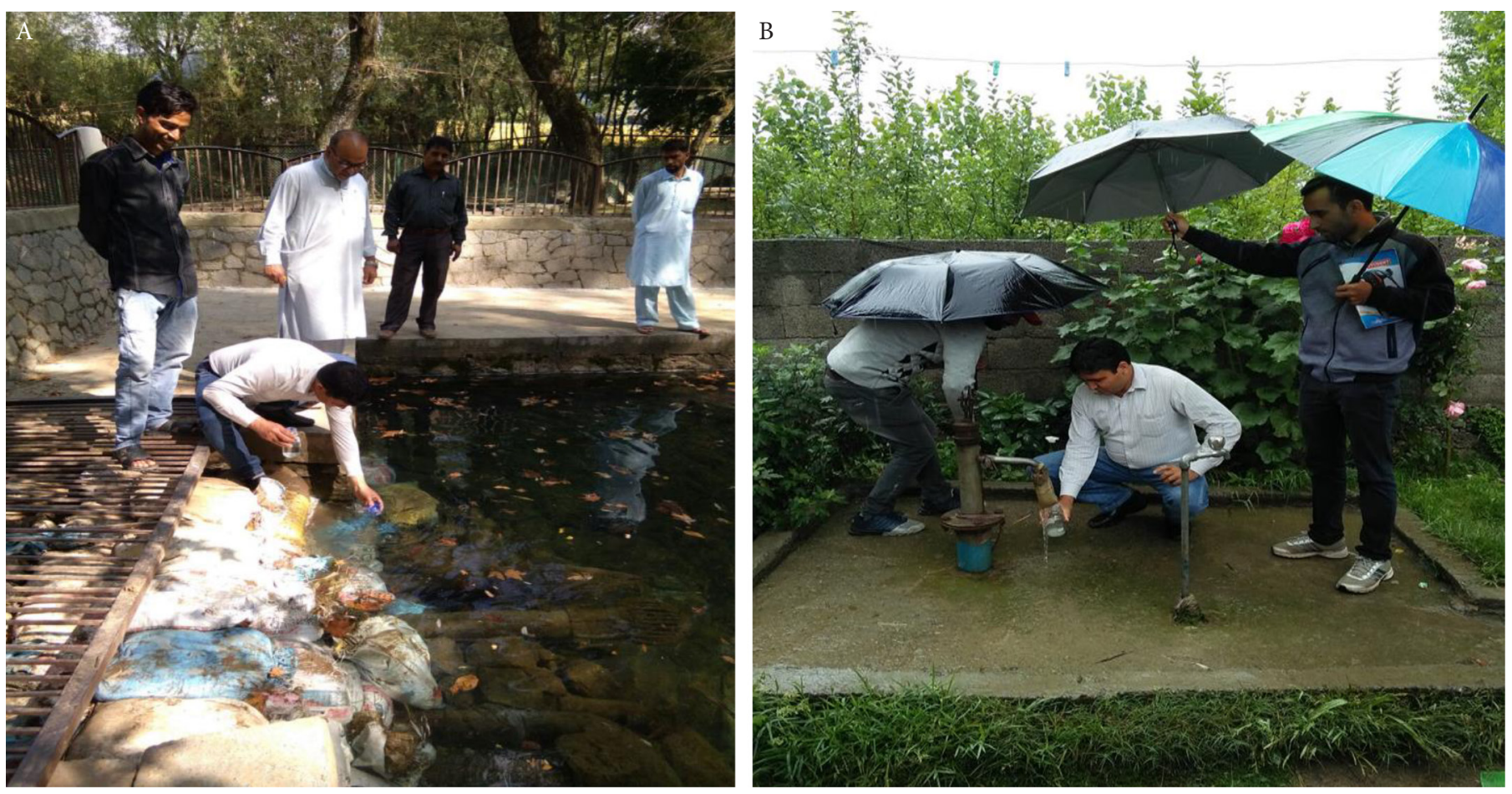

Figure 1 (A) Water sample collection at open source. (B) Water sample collection at a bore well (hand pump)

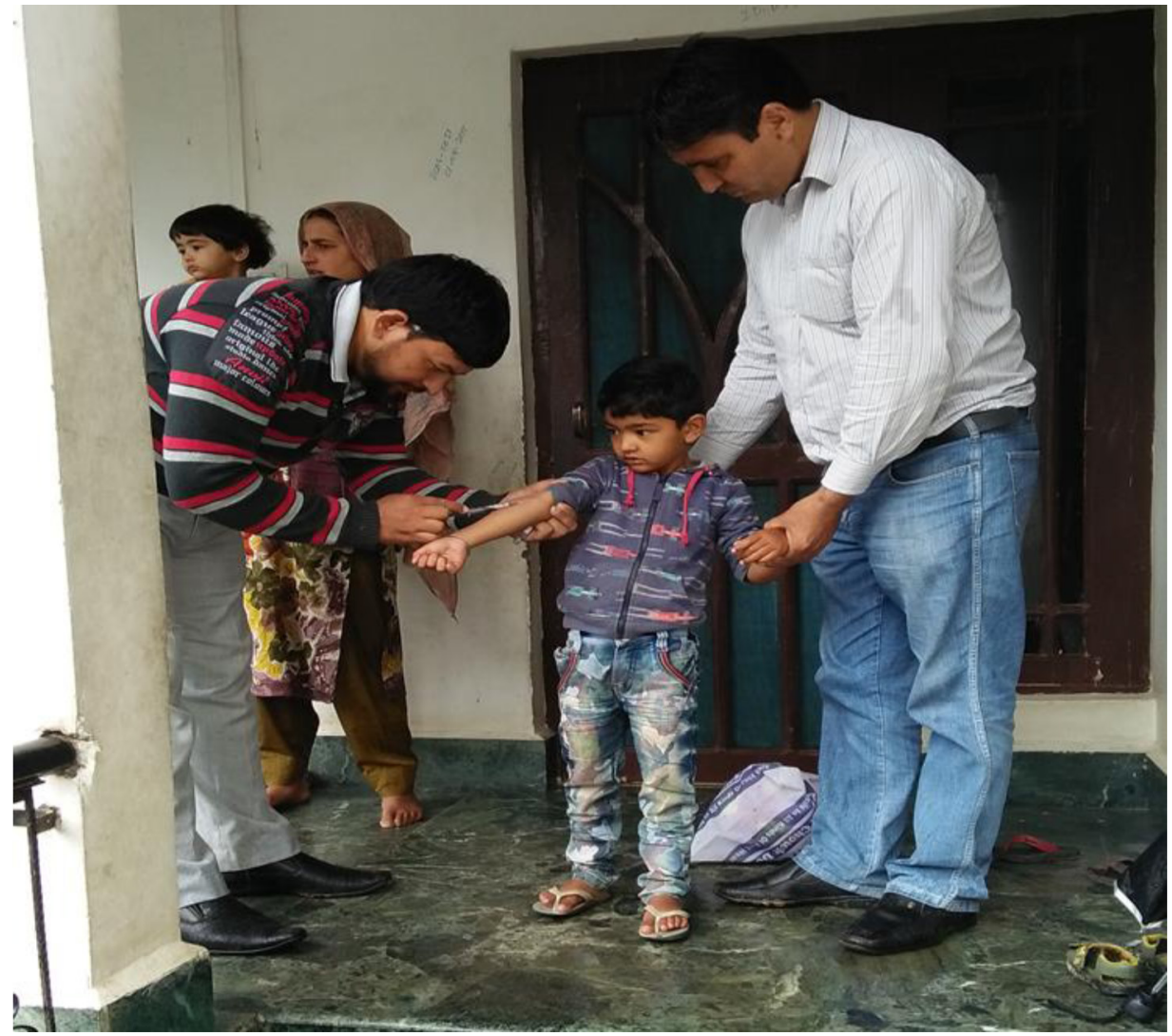

Figure 2 Collection of blood sample for hepatitis A in Kulgam 


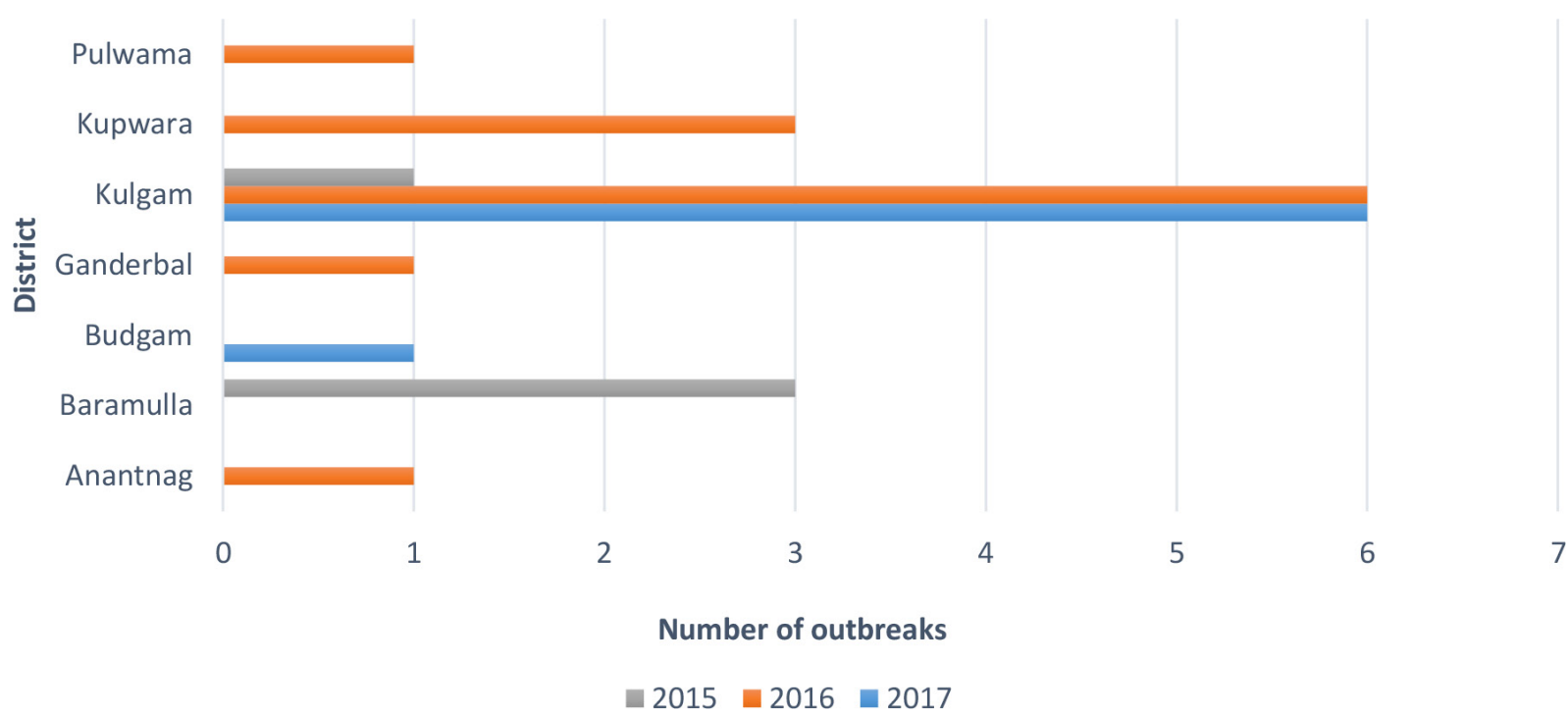

Figure 3 Number of hepatitis A and E outbreaks per district during 2015-2017

Table 1 Epidemiological data of hepatitis outbreaks during 2015-2017

\begin{tabular}{|c|c|c|c|c|c|c|c|c|}
\hline $\begin{array}{l}\text { No of } \\
\text { outbreaks }\end{array}$ & District & Area & Date & Disease & $\begin{array}{l}\text { No. of cases } \\
\qquad(\mathrm{M} / \mathrm{F})\end{array}$ & $\begin{array}{l}\text { Age group } \\
\text { (years) }\end{array}$ & $\begin{array}{l}\text { Blood samples } \\
\quad \text { (positive) }\end{array}$ & $\begin{array}{l}\text { Water samples } \\
\text { (unfit for human } \\
\text { consumption) }\end{array}$ \\
\hline \multicolumn{9}{|l|}{2015} \\
\hline 1 & Baramulla & Hanjiwara Bala & Jun 5, 2015 & Hep E & $40(31 / 9)$ & $21-57$ & $5 / 5$ & $2 / 2$ \\
\hline 2 & Baramulla & Barzulla, Kunzer & Jan 18, 2015 & Hep A & $20(11 / 9)$ & $4-53$ & $6 / 6$ & $3 / 4$ \\
\hline 3 & Baramulla & Dangiwacha & Sep 14, 2015 & Hep A & $63(39 / 24)$ & $5-13$ & $13 / 20$ & $3 / 4$ \\
\hline 4 & Kulgam & Nipora, Qazigund & Nov 16, 2015 & Hep E & $18(10 / 8)$ & $16-45$ & $2 / 10$ & $2 / 2$ \\
\hline \multicolumn{9}{|l|}{2016} \\
\hline 5 & Anantnag & Hingpora, Veerinag & Jan 13, 2016 & Hep A & $24(11 / 13)$ & $12-31$ & $4 / 8$ & $2 / 2$ \\
\hline 6 & Ganderbal & Ganiwan, Kangan & Jun 26, 2016 & Hep A & $8(4 / 4)$ & $3-10$ & $4 / 4$ & $0 / 2$ \\
\hline 7 & Kulgam & Chekbadwani, Qazigund & Dec 04, 2016 & Jaundice & $8(6 / 2)$ & $4-6$ & $0 / 5$ & $0 / 2$ \\
\hline 8 & Kulgam & Chekiwangund, Qazigund & Sep 26, 2016 & Hep E & $59(31 / 28)$ & $5-62$ & $13 / 13$ & $2 / 2$ \\
\hline 9 & Kulgam & Gaziabad, Yaripora & Oct 25,2016 & Hep E & $8(6 / 2)$ & $4-7$ & $4 / 6$ & 0 \\
\hline 10 & Kulgam & Tankipora, Yaripora & Jan 13,2016 & Hep E & $15(8 / 7)$ & $5-20$ & $3 / 5$ & 0 \\
\hline 11 & Kulgam & Banimulla, Kulgam & Jun 26, 2016 & Hep A & $19(9 / 10)$ & $5-8$ & $3 / 5$ & $2 / 2$ \\
\hline 12 & Kulgam & Matibug, Yaripora & Dec 04, 2016 & Hep A & $13(4 / 9)$ & $4-36$ & $3 / 13$ & $3 / 3$ \\
\hline 13 & Kupwara & Kunan, Trehgam & Sep 26, 2016 & Hep E & $11(6 / 5)$ & $17-42$ & $8 / 11$ & $2 / 2$ \\
\hline 14 & Kupwara & Hanga, Langate & Oct 25,2016 & Hep E & $16(7 / 9)$ & $5-42$ & $3 / 6$ & $3 / 3$ \\
\hline 15 & Kupwara & Budinambal, Kralpora & Jan 13,2016 & Hep E & $9(6 / 3)$ & $12-45$ & $3 / 3$ & $1 / 1$ \\
\hline 16 & Pulwama & Chandgam, Pulwama & Jun 26, 2016 & Hep A & $7(5 / 2)$ & $9-24$ & $1 / 4$ & $2 / 2$ \\
\hline \multicolumn{9}{|l|}{2017} \\
\hline 17 & Budgam & Watterhad, Khansahib & Oct 08,2017 & Hep A & $8(5 / 3)$ & $1-12$ & $3 / 8$ & $2 / 2$ \\
\hline 18 & Kulgam & Sadwari, Qazigund & Sep 21, 2017 & Hep A & $10(1 / 9)$ & $4-30$ & $3 / 5$ & $2 / 2$ \\
\hline 19 & & Ahmadabad & Jul 06, 2017 & Hep E & $8(2 / 6)$ & $15-65$ & $5 / 7$ & $3 / 3$ \\
\hline 20 & & Ujroo, Qazigund & Jun 21, 2017 & Hep A & $6(4 / 2)$ & $5-8$ & $6 / 6$ & $3 / 3$ \\
\hline 21 & & Baihama, Qazigund & Feb 22, 2017 & Hep E & $8(5 / 3)$ & $17-38$ & $2 / 7$ & $3 / 3$ \\
\hline 22 & & Shalipora, Kulgam & Aug 28, 2017 & Hep A & $9(6 / 3)$ & $16 \mathrm{~m}-17 \mathrm{y}$ & $6 / 6$ & $2 / 2$ \\
\hline 23 & & Kurigam, Qazigund & Mar 16, 2017 & Hep A & $6(2 / 4)$ & $7-18$ & $3 / 5$ & $0 / 2$ \\
\hline
\end{tabular}

Hep A, Hepatitis A; Hep E, Hepatitis E.

In 2016, collected data concerned five outbreaks of hepatitis A, six outbreaks of hepatitis E, and one cluster of cases with jaundice and negative blood samples for both HAV and HEV. The districts involved were Anantnag, Ganderbal, Kulgam, Kupwara, and Pulwama and the specific areas were Hingpora, Ganiwan, Chekbadwani, Gaziabad, Tankipora, Banimulla, Matibug, Kunan, Hanga, Budinambal, and Chandgam. The total number of cases registered was 197 (103 males and 94 females): 71 cases as hepatitis A, 118 cases as hepatitis E, and eight cases as seronegative jaundice. Of the 83 blood samples taken, 49 were found to be positive with seropositivity rates reaching $44 \%$ ( $15 / 34$ blood samples positive) for hepatitis A and 77\% (34/44 blood samples positive) for hepatitis E. Concerning the age group affected, three of the outbreaks mainly involved children younger than 10 years (range, 3-10 years), one 
outbreak involved children and young adolescents (range, 5-20 years), and the remaining outbreaks involved both children and adult population (range, 4-62 years). For water quality testing, 21 water samples were collected and tested, of which 15 were found unsatisfactory (i.e., unfit for human consumption), one sample was found suspicious and five samples were found satisfactory (i.e., fit for human consumption).

In 2017, the IDSP recorded seven outbreaks (five hepatitis A outbreaks and two hepatitis E outbreaks, which included 39 and 16 cases, respectively). Of the 55 cases studied, 25 were male and 30 were female patients. The districts affected were Budgam and Kulgam, specifically the areas of Watterhad, Sadwari, Ahmadabad, Ujroo, Baihama, Shalipora, and Kurigam. A total of 21/30 blood samples tested positive for HAV, whereas 7/14 blood samples tested positive for HEV. Although ages of the affected population ranged from 16 months to 65 years, most cases involved children (age $<18$ years). As far as water sampling is concerned, 17 samples were tested, which detected 13 samples with unsatisfactory quality and two with excellent quality. The results of the last two samples are still awaited.

\section{DISCUSSION}

For many years, India has been considered as one of the moderate to high endemic countries for hepatitis A and E infections; however, the recent socioeconomic development of the country has led to a mosaic of high and low endemic areas, suggesting that the country is now moderate endemic [6,7]. The anti-HAV level in Indian newborns has been reduced to a rate of 50-60\% compared with $100 \% 15$ years ago, and recent studies have shown that the age group of maximum exposure to HAV has shifted from children aged 6 months to 2 years to older children and young adolescents $[6,8]$, a fact that is also supported by the data presented in our study. In particular, most cases in our surveillance program involved children under the age of 18 , whereas some of the outbreaks also affected adult population. The district of Kulgam was the most affected, accounting for 13 outbreaks during 3-year monitoring period. By contrast, Kupwara and Baramulla, the second most affected regions, recorded only three outbreaks. The IDSP has also been recording various other outbreaks in Kulgam and other districts through epidemiological methods and diagnosing diseases based on clinical signs and symptoms [9].

Regarding seasonality, 13/23 outbreaks occurred during the monsoon (wet) season (i.e., June-October), which indicates the key role of hygiene, sanitary, and socioeconomic conditions in the transmission of hepatitis A and E viruses. In this spirit, water quality remains one of the most valuable factors affecting people's health and well-being and can, therefore, contribute to either the increase or the decrease (reductions) of hepatitis outbreaks. The crucial role of water as a source of contamination and transmission of hepatitis $A$ and $E$ as well as other diseases can be established based on our findings, in which at least one water sample was found to be unsatisfactory, according to the MPN method for counting coliform, in $16 / 21$ regions (rural areas) in which a hepatitis outbreak has been reported.

\section{CONCLUSION}

Hepatitis A and E viruses are transmitted mainly through the fecal-oral route due to fecal contamination of drinking water. As far as prevention is concerned, apart from vaccination for hepatitis A, adequate and clean water supplies along with thorough personal hygiene and sanitary measures play a major role in reducing outbreaks, considering that no specific antiviral therapy exists for either hepatitis A or E [10]. Thus, periodic surveillance of the HAV/HEV exposure pattern along with collaboration of public sectors to supply safe water and ensure safe sewage disposal may be of immense public health value. The data presented in this study indicate that infection with both the enteric hepatic viruses (HAV and HEV) is not infrequent and is mainly related to water quality in the areas studied. Therefore, our data will be useful for planning of future vaccination strategies and better sanitation programs in this part of the country seems essential.

\section{CONFLICTS OF INTEREST}

No conflicts of interest have been declared.

\section{REFERENCES}

[1] Yang K, LeJeune J, Alsdorf D, Lu B, Shum CK, Liang S. Global distribution of outbreaks of water-associated infectious diseases. PLoS Negl Trop Dis 2012;6;e1483.

[2] Arora D, Jindal N, Shukla RK, Bansal R. Water borne hepatitis A and hepatitis E in Malwa region of Punjab, India. J Clin Diagn Res 2013;7;2163-6.

[3] Kotwal A, Singh H, Verma AK, Gupta RM, Jain S, Sinha S, et al. A study of hepatitis A and E virus seropositivity profile amongst young healthy adults in India. Med J Armed Forces India $2014 ; 70 ; 225-9$.

[4] World Health Organization (WHO). Hepatitis A [Internet]. Geneva: WHO; 2017. Available from: http://www.who.int/mediacentre/factsheets/fs328/en/ [cited October 24, 2017]

[5] Kumar T, Shrivastava A, Kumar A, Laserson KF, Narain JP, Venkatesh S, et al. Viral hepatitis surveillance-India, 2011-2013. MMWR Morb Mortal Wkly Rep 2015;64;758-62.

[6] Barzaga BN. Hepatitis A shifting epidemiology in South-East Asia and China. Vaccine 2000;18 Suppl 1;S61-S4.

[7] Jacobsen KH, Wiersma, ST. Hepatitis A virus seroprevalence by age and world region, 1990 and 2005. Vaccine 2010;28;6653-57.

[8] Mathur P, Arora NK. Epidemiological transition of hepatitis A in India: issues for vaccination in developing countries. Indian J Med Res 2008;128;699-704.

[9] Kadri SM, Rehman SU, Rehana K, Brady AH, Chattu VK. Should mumps be higher up on the public health agenda in India? A concern for global health security. Med Sci (Basel) 2018;6;E62.

[10] WHO. Water-related diseases [Internet]. Geneva: WHO; 2001. Available from: http://www.who.int/water_sanitation_health/ diseases-risks/diseases/hepatitis/en/ [cited February 25, 2018]. 